\title{
Percutaneous endoscopic gastrostomy placement during pregnancy in the critical care setting
}

A 37-year-old woman with a past medical history of untreated hypertension presented with unresponsiveness at 28 weeks of gestation. Computed tomography (CT) revealed a pontine hemorrhage with massive edema. After 2 weeks of nasogastric feeding, the patient received a percutaneous endoscopic gastrostomy (PEG) tube. At 31 weeks, cesarean section was performed and a 1660 -g preterm infant was delivered. The patient continued on PEG feeding and slight neurological improvement was seen.

Optimal nutritional requirements are critical in the intensive care unit as evidenced by the critical care and pancreatitis guidelines [1]. During pregnancy, optimal nutrition is essential in order to minimize maternal and neonatal morbidity [2]. Longterm nasogastric feeding is limited by patient tolerability and nasal septal necrosis. The long-term side effects of total parenteral nutrition limit its usage during pregnancy [3]. Thus, PEG becomes an impor- tant option for long-term enteral feeding [4]. However, concerns about uterine damage, fetal injury, premature labor, and infections have restricted the application of PEG tube placement in pregnant women. Our study reviews the safety and feasibility of PEG tube placement in pregnancy in the critical care setting.

There were no major complications with PEG tube placement in the 11 reported cases in the literature [4-11], as well as in our case ( Table 1 ). PEG enteral nutritional support was provided for an average of 14 weeks in the literature. During pregnancy, PEG tube placement is a feasible procedure for optimal enteral nutrition in the critical care setting. It is also feasible to perform PEG tube placement in the third trimester of pregnancy. Special precautions ( $\bullet$ Table 2 ) are critical for PEG tube placement during pregnancy, and knowledge of these precautions is essential.
In conclusion, a review of the literature clearly shows that the risk of malnutrition in pregnancy greatly exceeds the risk of PEG placement.

\section{Competing interests: None}

\section{Endoscopy_UCTN_Code_TTT_1AO_2AC}

\section{Senadhi ${ }^{1}$, J. Chaudhary ${ }^{1}$, S. Dutta ${ }^{2}$}

1 Johns Hopkins University/Sinai Hospital Program in Internal Medicine, Department of Internal Medicine, Sinai Hospital, Baltimore, Maryland, USA

2 Johns Hopkins University/Sinai Hospital Program and the University of Maryland School of Medicine, Division of Gastroenterology, Sinai Hospital, Baltimore, Maryland, USA

\section{References}

1 Zarbock SD, Steinke D, Hatton J et al. Successful enteral nutritional support in the neurocritical care unit. Neurocrit Care 2008; 9: 210-216

2 Villar J, Merialdi M, Gülmezoglu AM et al. Nutritional interventions during pregnancy for the prevention or treatment of maternal morbidity and preterm delivery: an overview of randomized controlled trials. J Nutr 2003; 133: 1606S-1625S

3 Wong M, Apodaca CC, Markenson MG, Yancey $M$. Nutrition management in a pregnant

Table 1 Features of cases where a percutaneous endoscopic gastrostomy (PEG) tube was placed during pregnancy.

\begin{tabular}{|c|c|c|c|c|c|c|c|c|}
\hline Reference & $\begin{array}{l}\text { Patient's } \\
\text { age, } \\
\text { years }\end{array}$ & $\begin{array}{l}\text { Gestational } \\
\text { age at pre- } \\
\text { sentation, } \\
\text { weeks }\end{array}$ & $\begin{array}{l}\text { Indication for } \\
\text { PEG tube }\end{array}$ & $\begin{array}{l}\text { Duration of } \\
\text { nutritional } \\
\text { support, } \\
\text { weeks }\end{array}$ & $\begin{array}{l}\text { Delivery type/ } \\
\text { gestational } \\
\text { age, weeks }\end{array}$ & $\begin{array}{l}\text { Birth } \\
\text { weight, } \\
\text { g }\end{array}$ & $\begin{array}{l}\text { Maternal and } \\
\text { fetal outcome }\end{array}$ & $\begin{array}{l}\text { Special precau- } \\
\text { tions taken }\end{array}$ \\
\hline $\begin{array}{l}\text { Koh \& } \\
\text { Lipkin } 1993\end{array}$ & 24 & 13 & $\begin{array}{l}\text { Motor vehicle } \\
\text { accident with } \\
\text { coma }\end{array}$ & 24 & $\begin{array}{l}\text { Cesarean } \\
\text { section/37 }\end{array}$ & 3680 & $\begin{array}{l}\text { Mother } \\
\text { improved; } \\
\text { baby well }\end{array}$ & п.а. \\
\hline \multirow[t]{2}{*}{$\begin{array}{l}\text { Shaheen } \\
\text { et al. } 1997\end{array}$} & 19 & 17 & $\begin{array}{l}\text { Anorexia and } \\
\text { odynophagia } \\
\text { due to esophagi- } \\
\text { tis }\end{array}$ & 5 & $\begin{array}{l}\text { Natural vaginal } \\
\text { delivery/24 }\end{array}$ & 2440 & $\begin{array}{l}\text { Mother } \\
\text { improved; } \\
\text { baby well }\end{array}$ & $\begin{array}{l}\text { - Ultrasound guid- } \\
\text { ance to define the } \\
\text { dome of the uterus } \\
\text { - Repeated adjust- } \\
\text { ments were re- } \\
\text { quired to avoid } \\
\text { pressure necrosis }\end{array}$ \\
\hline & 34 & 24 & $\begin{array}{l}\text { Congenital } \\
\text { myotonic } \\
\text { dystrophy and } \\
\text { mental retarda- } \\
\text { tion }\end{array}$ & n. a. & $\begin{array}{l}\text { Cesarean } \\
\text { section/30 }\end{array}$ & 1080 & $\begin{array}{l}\text { Both mother } \\
\text { and baby well }\end{array}$ & $\begin{array}{l}\text { - Same as above } \\
\text { - Abdominal binder } \\
\text { over PEG tube to } \\
\text { guard against acci- } \\
\text { dental dislodge- } \\
\text { ment }\end{array}$ \\
\hline \multirow[t]{2}{*}{$\begin{array}{l}\text { Godil \& } \\
\text { Chen } 1998\end{array}$} & 18 & 16 & $\begin{array}{l}\text { Anorexia } \\
\text { nervosa }\end{array}$ & 10 & $\begin{array}{l}\text { Natural vaginal } \\
\text { delivery/39 }\end{array}$ & 2782 & $\begin{array}{l}\text { Both mother } \\
\text { and baby well }\end{array}$ & \multirow{2}{*}{$\begin{array}{l}\text { - Antibiotic pro- } \\
\text { phylaxis } \\
\text { - Sedation with } \\
\text { midazolam and } \\
\text { intravenous } \\
\text { meperidine }\end{array}$} \\
\hline & 14 & 29 & $\begin{array}{l}\text { Hyperemesis } \\
\text { gravidarum }\end{array}$ & 10 & $\begin{array}{l}\text { Natural vaginal } \\
\text { delivery/39 }\end{array}$ & 3000 & $\begin{array}{l}\text { Both mother } \\
\text { and baby well }\end{array}$ & \\
\hline
\end{tabular}


Table 1 Features of cases where a percutaneous endoscopic gastrostomy (PEG) tube was placed during pregnancy.

\begin{tabular}{|c|c|c|c|c|c|c|c|c|}
\hline Reference & $\begin{array}{l}\text { Patient's } \\
\text { age, } \\
\text { years }\end{array}$ & $\begin{array}{l}\text { Gestational } \\
\text { age at pre- } \\
\text { sentation, } \\
\text { weeks }\end{array}$ & $\begin{array}{l}\text { Indication for } \\
\text { PEG tube }\end{array}$ & $\begin{array}{l}\text { Duration of } \\
\text { nutritional } \\
\text { support, } \\
\text { weeks }\end{array}$ & $\begin{array}{l}\text { Delivery type/ } \\
\text { gestational } \\
\text { age, weeks }\end{array}$ & $\begin{array}{l}\text { Birth } \\
\text { weight, } \\
\text { g }\end{array}$ & $\begin{array}{l}\text { Maternal and } \\
\text { fetal outcome }\end{array}$ & $\begin{array}{l}\text { Special precau- } \\
\text { tions taken }\end{array}$ \\
\hline \multirow[t]{2}{*}{$\begin{array}{l}\text { Serrano } \\
\text { et al. } 1998\end{array}$} & 25 & 11 & $\begin{array}{l}\text { Hyperemesis } \\
\text { gravidarum }\end{array}$ & 18 & $\begin{array}{l}\text { Natural vaginal } \\
\text { delivery/40 }\end{array}$ & 4000 & $\begin{array}{l}\text { Both mother } \\
\text { and baby well }\end{array}$ & \multirow[t]{2}{*}{$\begin{array}{l}\text { - Radiograph with } \\
\text { pelvic shielding to } \\
\text { verify the position } \\
\text { of jejunal tube }\end{array}$} \\
\hline & 25 & 15 & $\begin{array}{l}\text { Hyperemesis } \\
\text { gravidarum }\end{array}$ & 20 & $\begin{array}{l}\text { Natural vaginal } \\
\text { delivery/36 }\end{array}$ & 2750 & $\begin{array}{l}\text { Both mother } \\
\text { and baby well }\end{array}$ & \\
\hline $\begin{array}{l}\text { O'Connell } \\
\text { et al. } 2000\end{array}$ & 24 & 11 & $\begin{array}{l}\text { Chronic } \\
\text { malnutrition }\end{array}$ & & $\begin{array}{l}\text { Cesarean } \\
\text { section/33 }\end{array}$ & 1620 & $\begin{array}{l}\text { Both mother } \\
\text { and baby well }\end{array}$ & n.a. \\
\hline $\begin{array}{l}\text { Wejda } \\
\text { et al. } 2003\end{array}$ & 41 & 8 & $\begin{array}{l}\text { Apallic } \\
\text { syndrome }\end{array}$ & 19 & $\begin{array}{l}\text { Cesarean } \\
\text { section/27 }\end{array}$ & 820 & $\begin{array}{l}\text { Mother con- } \\
\text { tinued on nutri- } \\
\text { tion therapy; } \\
\text { baby well }\end{array}$ & n.a. \\
\hline Irving 2004 & 32 & 17 & $\begin{array}{l}\text { Severe hyper- } \\
\text { emesis gravi- } \\
\text { darum }\end{array}$ & 18 & $\begin{array}{l}\text { Cesarean } \\
\text { section/35 }\end{array}$ & 2300 & $\begin{array}{l}\text { Both mother } \\
\text { and baby well }\end{array}$ & $\begin{array}{l}\text { - Generalized } \\
\text { anesthesia with } \\
\text { antibiotic } \\
\text { - Continuous ultra- } \\
\text { sound guidance } \\
\text { and monitoring } \\
\text { of fetus }\end{array}$ \\
\hline $\begin{array}{l}\text { Fedorka } \\
2004\end{array}$ & 34 & 10 & $\begin{array}{l}\text { Motor vehicle } \\
\text { accident }\end{array}$ & 24 & $\begin{array}{l}\text { Cesarean } \\
\text { section/34 }\end{array}$ & 2608 & $\begin{array}{l}\text { Mother con- } \\
\text { tinued on vege- } \\
\text { tative state } \\
\text { support; baby } \\
\text { well }\end{array}$ & n.a. \\
\hline $\begin{array}{l}\text { Senadhi, } \\
\text { Chaudhary } \\
\text { \& Dutta } \\
2010 \\
\text { (current } \\
\text { report) }\end{array}$ & 37 & 27 & $\begin{array}{l}\text { Intracranial } \\
\text { bleed (pontine } \\
\text { hemorrhage) }\end{array}$ & 2 & $\begin{array}{l}\text { Cesarean } \\
\text { section/31 }\end{array}$ & 1660 & $\begin{array}{l}\text { Mother con- } \\
\text { tinued on nutri- } \\
\text { tion therapy } \\
\text { with slight } \\
\text { neurological } \\
\text { improvement; } \\
\text { baby well }\end{array}$ & $\begin{array}{l}\text { - Ultrasound guid- } \\
\text { ance with fundal } \\
\text { monitoring before } \\
\text { the procedure } \\
\text { - Continuous fetal } \\
\text { monitoring by an } \\
\text { obstetric nurse } \\
\text { - Operating room } \\
\text { ready for precipi- } \\
\text { tated labor }\end{array}$ \\
\hline
\end{tabular}

n. a., not applicable.

Table 2 Recommendations for percutaneous endoscopic gastrostomy (PEG) tube placement during pregnancy.

Recommendations during pregnancy
Ultrasound to define the dome of the uterus before the procedure
Ultrasound indentation and transillumination displaying PEG can be separated from the rib cage and the
uterus
Continuous fetal monitoring by an obstetric nurse throughout the procedure
Operating room ready for precipitated labor during PEG placement
Monitor fetal growth and development through ultrasound, especially in second and third trimester
Careful monitoring of the tension on the external bumper of the PEG to avoid excess external bumper pres-
sure as the uterus enlarges
Repeated adjustments are needed to avoid pressure necrosis from the tension in the area from the internal
and external bumpers
Procedural sedation can be safely achieved with propofol (pregnancy category B)

comatose patient. Nutr Clin Pract 1997; 12: $63-67$

4 Koh ML, Lipkin EW. Nutrition support of a pregnant comatose patient via percutaneous endoscopic gastrostomy. JPEN J Parenter Enteral Nutr 1993; 17: 384-387

5 Shaheen NJ, Crosby MA, Grimm IS, Isaacs K. The use of percutaneous endoscopic gastrostomy in pregnancy. Gastrointest Endosc 1997; 46: $564-565$

6 Godil A, Chen YK. Percutaneous endoscopic gastrostomy for nutrition support in pregnancy associated with hyperemesis gravi- darum and anorexia nervosa. JPEN J Parenter Enteral Nutr 1998; 22: 238-241

7 Serrano P, Velloso A, García-Luna PP et al. Enteral nutrition by percutaneous endoscopic gastrojejunostomy in severe hyperemesis gravidarum: a report of two cases.

8 O'Connell M, Wilson O, Masson E, Lindau S. Pregnancy outcome in a patient with chronic malnutrition. Hum Reprod 2000; 15: $2443-2445$

9 Wejda BU, Soennichsen B, Huchzermeyer H et al. Successful jejunal nutrition therapy in a Clin Nutr 1998; 17: 135-139 pregnant patient with apallic syndrome. Clin Nutr 2003; 22: 209-211

10 Irving PM, Howell RJ, Shidrawi RG. Percutaneous endoscopic gastrostomy with a jejunal port for severe hyperemesis gravidarum. Eur J Gastroenterol Hepatol 2004; 16: 937 939

11 Fedorka P, Sullivan J. Case report: persistent vegetative state in pregnancy. Top Emerg Med 2004; 26: 49-51

Bibliography

DOI $10.1055 / \mathrm{s}-0030-1256052$

Endoscopy 2010; 42: E358 - E359

(c) Georg Thieme Verlag KG Stuttgart - New York . ISSN 0013-726X

\section{Corresponding author \\ Dr. Viplove Senadhi}

Johns Hopkins University/Sinai Hospital Program in Internal Medicine, Sinai Hospital

2401 West Belvedere Avenue

Baltimore, Maryland 21215, USA

Fax: +1-678-623-5999

vsenadhi@hotmail.com 